\title{
THE EFFECT OF DEEP INBREATHING ON LEAD III OF THE ELECTROCARDIOGRAM
}

\author{
BY \\ WILLIAM EVANS \\ House Physician to Dr. John Parkinson, 1927; Paterson M.O. and Chief Assistant to the Cardiac Department, London \\ Hospital, 1933 and 1934; Assistant Physician to the Cardiac Department, London Hospital, $1938-48$.
}

Received January 17, 1951

The influence of the position of the heart on the form of the electrocardiogram has been known for close on half a century. It was discussed by Waller (1913 and 1914) and more fully by Einthoven et al. (1913), but the bearing of this fact on the diagnosis of heart disease has not received much attention. The effects of deep inbreathing on the tracing are found to be common, especially in lead III, showing that this lead may deceive if studied without regard to the lie of the heart in the chest. It was decided to test the effect of deep inspiration on lead III, both in health and in patients with heart disease, and the results of such observations are here described.

\section{LEAD IIIR IN HEALTH}

Since respiration plays a part in the production of the tracing, the designation IIIR is proposed for this lead where $\mathbf{R}$ stands for respiration. The lead was first specially examined in 200 healthy adults: none had symptoms of heart disease and none had abnormal signs on routine clinical and radiological examination; subjects under 20 years of age were excluded. The three limb leads were recorded in the semi-recumbent position; lead IIIR was then taken during halted respiration at the end of deep inspiration; the bipolar chest leads, CR1, CR4, and CR7, were also recorded; and unipolar limb lead VF was often added. In no single instance among the 200 cases was lead IIIR identical with lead III; sometimes the form of the curve was noticeably altered, but sometimes the change was confined to two of the primary waves or even to a single wave.

Naturally, the rate was often affected, usually quickening at the start of inspiration, but soon becoming slower even before expiration commenced. In five cases extrasystoles appeared in IIIR and in three these were present in III but absent in IIIR (Fig. 1); in another case deep inspiration always induced a short bout of paroxysmal tachycardia.

It is convenient to describe the changes that took place, in the case of $P, Q, R, S$, and $T$ waves, and of the $S-T$ segment in turn. As the P-R period was never abnormally prolonged it was not expected to change noticeably during deep inbreathing, nor did it.

The $P$ wave. P III was upright in 173, flat or isoelectric in 4, and inverted in 23 cases. In those where the P was upright in III, it became taller in IIIR in 109 , unchanged in 60 , and less tall in 4 subjects with a centrally placed heart on radiological examination. In the four with a flat P III, it was taller in IIIR in one and unchanged in the other three. Among the 23 with an inverted $P$ III, it was less inverted, flat, or upright in IIIR in 22, and unchanged once.

The $Q$ wave. The initial deflection of the QRS III complex was directed downwards (Q) in 69 cases and upwards (R) in the remaining 131.

Where Q III was present, it was deep (greater than $2 \mathrm{~mm}$.) in 16, and less than $2 \mathrm{~mm}$. in the remaining 53; in this second group it remained unchanged in IIIR in 35 and disappeared in 18 cases. In the 16 cases in the first group it was absent in IIIR in 7, and it was also less deep in the other 
9 cases. Thus, a Q III was shown to be without significance in 25 out of 69 cases, for it was not present in IIIR. Videla (1939 and 1942) in a careful study of this subject found that Q III became less deep in cases with a high diaphragm during inspiration which depressed the diaphragm. Lyle (1944) stated that in some cases without cardiac pain where there was no $Q$ wave in the unipolar lead VF, and where presumably a $Q$ in lead III was a positional effect, it could not be made to disappear by deep inbreathing; she concluded that its failure to disappear was not incontrovertible proof of a coronary origin.

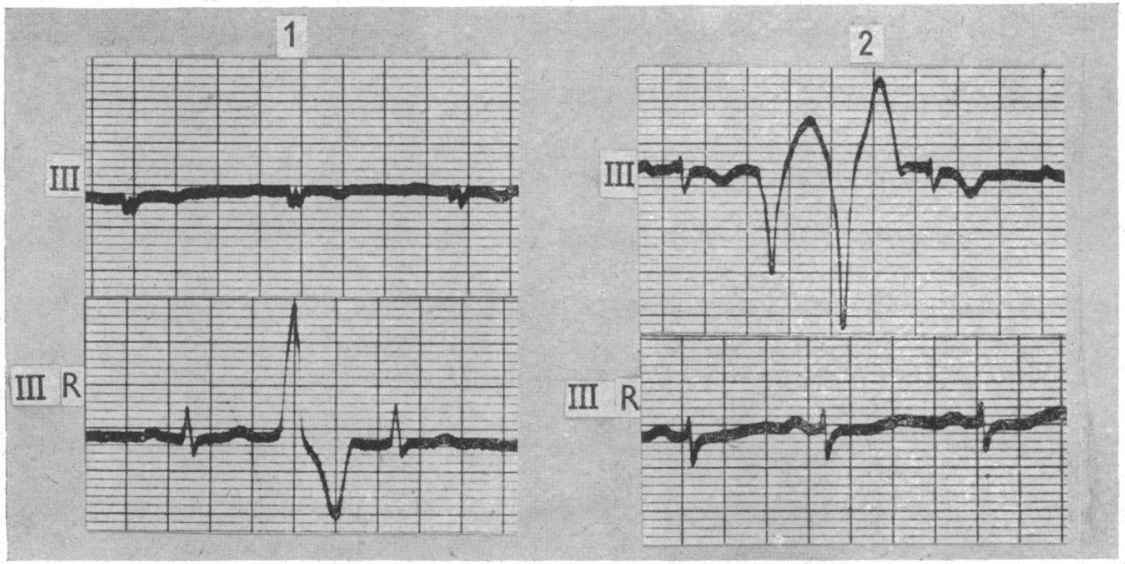

FIG. 1

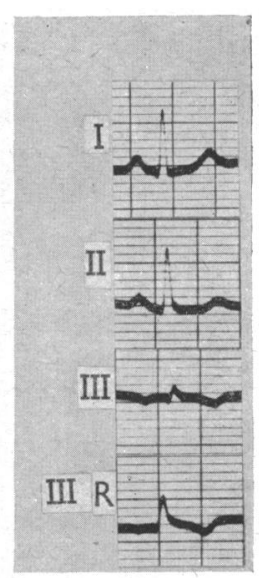

FIG. 2

FIG. 1.-Extrasystoles induced by deep inspiration in Case 1, and anulled in Case 2 . FIG. 2.-High diaphragm in pregnancy. $T$ in IIIR inverted as in III.

In none of my healthy cases did a $Q$ wave that was deep in III remain deep in IIIR, neither did a $Q$ wave ever become exaggerated in IIIR, nor did a $Q$ appear in IIIR if it had been absent in III.

The $R$ wave. In each of the 11 cases where $R$ was absent in III it appeared in IIIR. In the remaining 189 where $R$ III was present, it became taller in IIIR in 137, remained unchanged in 47, and became less tall in 5 ; in one of these, the $S$ wave became deeper at the same time.

The $S$ wave. S III was absent in 90 and present in the remaining 110 cases. In the former group it remained absent in IIIR in all but two, and in these the heart was vertical. In the second group with S III present, it became less deep in 78 and in 34 of these it disappeared; it remained unchanged in 14 and in 18 it was deeper than in lead III.

The $T$ wave. Norris and Massie (1947) said there was a tendency, in health, for inspiration to increase the positivity of all deflections, including the $\mathrm{T}$ wave. In my series $\mathrm{T}$ III was upright in 107 , flat in 25 , and inverted in 68 of the 200 cases. In the first group of 107 cases $T$ was taller in IIIR in 91, and was unchanged in 16. In all of the second group of 25 cases the T in IIIR became upright. In the third group of 68 cases the inverted $T$ in lead III became upright in IIIR in 42 and less inverted in 25 cases; in one woman six months' pregnant, the inverted $T$ wave remained unchanged in IPIR (Fig. 2).

The $S-T$ segment. There was no change in the S-T segment in IIIR from that in III in 197 cases. In two of the remaining three cases it became raised in IIIR and in one of these it was slightly depressed in III. In only one subject did the S-T segment become depressed in IIIR and this was a man of 80 years of age who appeared to be a healthy subject but was subject to paroxysmal tachycardia.

Code of normality for lead IIIR. The foregoing analysis of the IIIR cardiogram has shown the common changes expected to take place in the tracing in health (Fig. 3) and has established what is the deviation from the normal pattern that tells of the presence of heart disease; such changes 
affect the $\mathrm{Q}$ and $\mathrm{T}$ waves and the $\mathrm{S}-\mathrm{T}$ segment; they are here described for each and deduced from the findings in this the control group of 200 healthy subjects.

A $Q$ wave of $2 \mathrm{~mm}$. or under in lead III may persist in IIIR in health, but a $Q$ deeper than $2 \mathrm{~mm}$. is never found in the healthy adult. Again, a $Q$ in III never deepens in IIIR, nor appears in IIIR when absent in III.

An upright $T$ wave in III never lessens in IIIR, while a flat or inverted $T$ rises significantly in IIIR; rarely, failure of the $T$ wave to rise may be met with in health if the diaphragm for any reason does not move downwards on inbreathing.

Depression of the $S-T$ segment in IIIR is a change not met with in health, and in the meantime such changes should be accepted as evidence of heart disease if not due to digitalization.

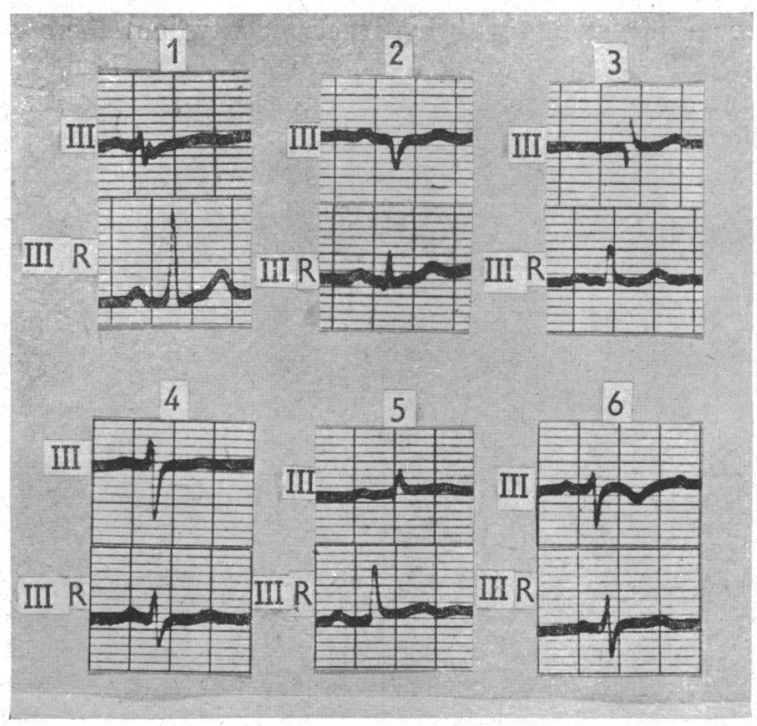

Fig. 3

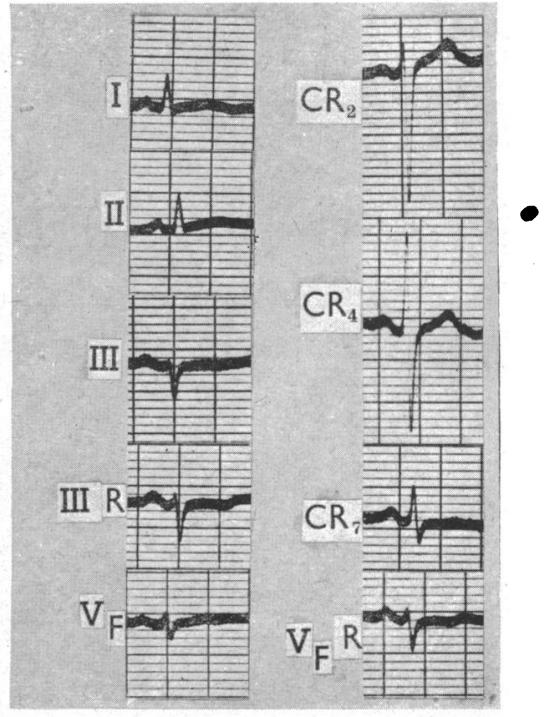

Fig. 4

FIG. 3.-Changes in IIIR in health. (1) $P$ taller. (2) $Q$ shorter. (3) $Q$ absent. (4) $R$ taller and $S$ shorter. (5) $T$ taller. (6) Inverted $T$ in III upright in IIIR.

FIG. 4.-Cardiac infarction. Flat $\mathrm{T}$ in III and VF inverted in IIIR and VFR.

Comparison of lead IIIR and VF. Myers and Oren (1945) found a Q wave in VF in 8 out of 25 healthy subjects who showed a.Q III. Obvious T inversion in VF is not as common a finding as in III because in the unipolar lead the influence of the electrical potential at the left leg, although present is less obvious. This investigation has shown that such a failing in III is invariable, for in the 200 healthy subjects assembled as a control group, leads IIIR and III were different in some - detail every time. Like lead III, VF is altered by deep inbreathing (Fig. 4 and 5). Thus VF does not give information comparable with that obtained from recording leads III and IIIR, and it is necessary to repeat VF during deep inbreathing (VFR) to obtain a cardiogram free from the effects of the natural uplift of the heart by the diaphragm. Even then, the change taking place in IIIR when the $\mathbf{T}$ wave has been obviously deformed in III in health is so striking as to provide valuable information which is not gained from a comparison of VF and VFR because of the minimal change which such a combination of leads shows.

\section{Lead IIIR in Heart Disease}

Having determined the pattern of the IIIR cardiogram in health, its form was next studied in the common kinds of heart disease, especially having in mind the help it might give in the detection 
of coronary artery disease before obvious changes showed in the cardiogram habitually used in the investigation of such cases.

Thus, lead IIIR was examined in 200 patients, 60 of them with left and 10 with right ventricular preponderance, 20 with left and 20 with right bundle branch block; and 77 with cardiac infarction. The remaining 13 had chest pain and lead IIIR provided the only cardiographic abnormality that might indicate coronary artery disease, the presence of which was proved by unequivocal evidence of cardiac infarction in other leads on previous or subsequent occasions.

\section{Left Ventricular Preponderance}

Two groups of patients were used to observe the behaviour of this lead in left ventricular preponderance, namely, those with hypertension ( 40 cases) and those with aortic valvular disease ( 20 cases).

In hypertension. Lead IIIR was examined in 40 patients with hypertension in whom there was left ventricular preponderance with depression of the $T$ wave and of the S-T segment in leads $I$ and CR7. It is known that this cardiographic pattern may often mask the changes that are the outcome of cardiac infarction, and IIIR was specially examined for the contribution it might make to help such a diagnosis. It was for this reason that the 40 patients were divided into two groups-20 patients with hypertension and without chest pain, and the other 20 with pain that subjectively was characteristically cardiac. These two groups will be considered separately.

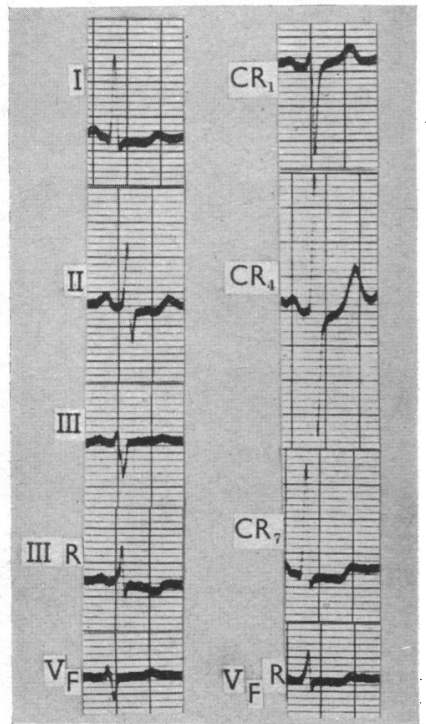

FIG. 5

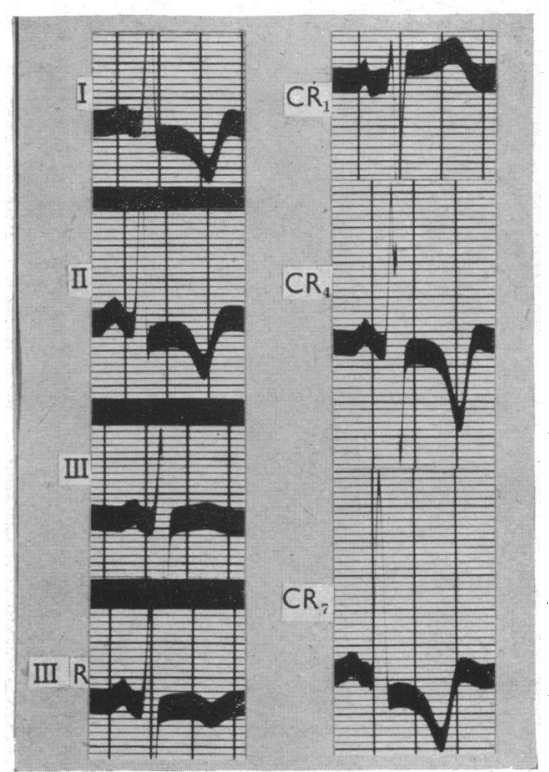

Fig. 6

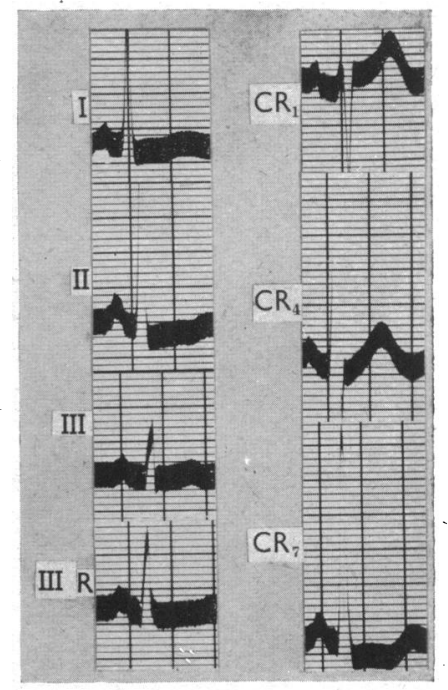

FIG. 7

Fig. 5.-Cardiac infarction. Upright $T$ in III and VF inverted in IIIR and VFR.

FIG. 6.- Hypertension without cardiac pain. Inversion of $T$ in IIIR suggests coronary arterial disease.

Fig. 7.-Nephritic hypertension without cardiac pain. Flat $T$ in IIIR suggests coronary arterial disease, and this was found at necropsy (see Fig. 8).

Hypertension without pain. There was depression of the S-T segment with lowering of the $\mathrm{T}$ wave in leads $I$ and CR7 in every case, but in only two was there deep and sharp inversion of the $T$ wave. The changes were always a little more noticeable in CR7 than in I. In only two was there inversion of the $T$ in CR4 and in both this change was less than in CR7.

In three of the cases the limb leads showed changes expected in posterior cardiac infarction, and lead IIIR showed depression of the $S-T$ segment and of the $T$ wave in each of these. In five others such changes in IIIR (Fig. 6, 7, and 8) provided the only cardiographic abnormality that might be evidence of underlying 


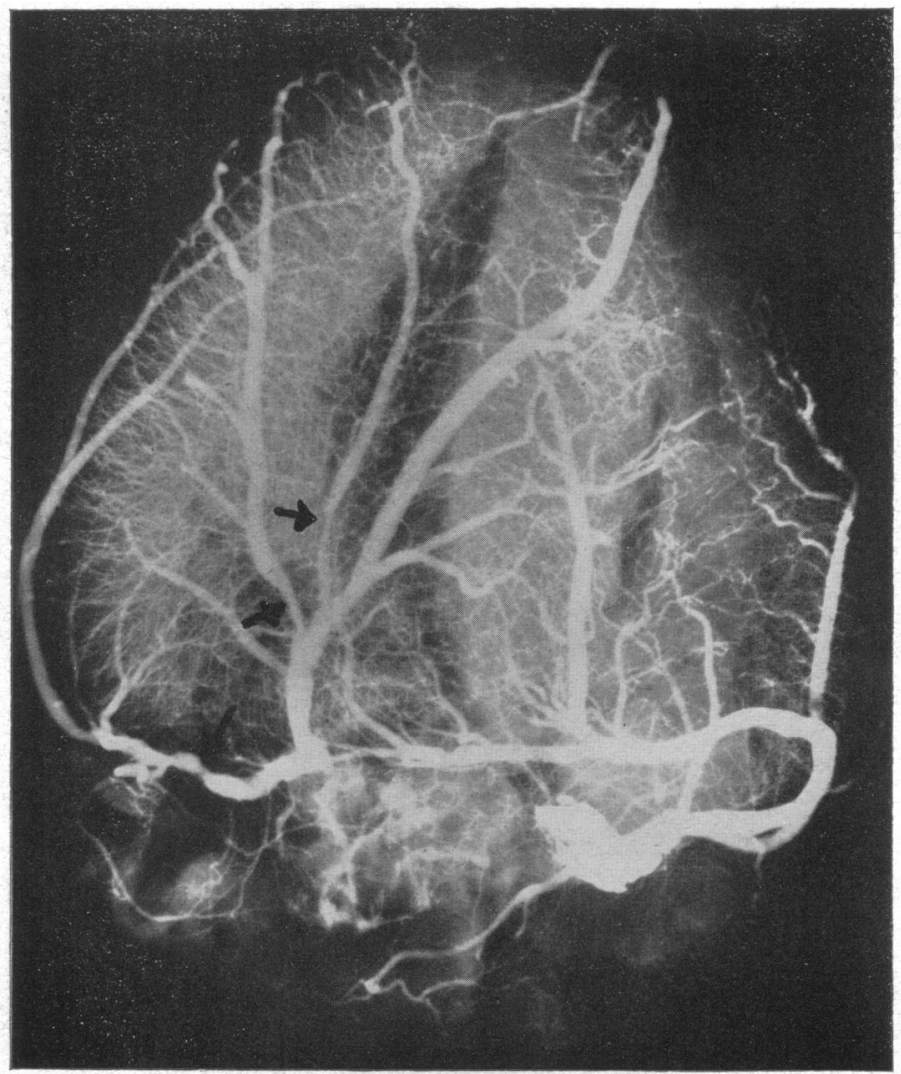

FIG. 8.-Coronary arterial disease in nephritic hypertension (Fig. 7). Arrows indicate sites of great narrowing.

coronary artery disease, and in two of these necropsy confirmed this; the other three patients are still alive. In the remaining 12, no signs in IIIR or any other lead were present to suggest the addition of coronary artery trouble to the existing hypertensive heart disease.

Hypertension with cardiac pain. There was depression of the S-T segment in leads I and CR7 in each of the 20 cases, together with a lowering of the $T$ wave, which in 9 cases was in the form of a deep inversion; such deep inversion was commoner in this group, for only two of the patients without pain showed it. Again, the inversion of T in CR4 was commoner ( 9 against 2 ) in this group where pain was an added symptom, and in 8 of them the $T$ inversion was greater in CR4 than in CR7. In another 7, evidence of posterior infarction was present in the limb leads II and III.

In each of the 20 patients an abnormal $Q$ wave (in five cases) and/or depression of the $S-T$ segment and the $T$ wave (in 18 cases) were present in lead IIIR (Fig. 9), indicating the presence of coronary arterial disease, if not of cardiac infarction as well.

A comparison of the two groups of patients with hypertension has allotted an importance to lead IIIR as a means of detecting coronary arterial disease when the signs of it may be masked by the cardiographic pattern of left ventricular preponderance from hypertension. The findings suggest that lead IIIR may also help to estimate the prognosis in a patient with hypertensive heart disease. In the cardiographic diagnosis of cardiac infarction in the presence of hypertension it may be said, therefore, that such can be presumed if some or all of the following findings are present; changes in the limb leads characteristic of infarction; deep inversion of the $T$ wave in the absence of a very tall $R$ wave (Shillingford and Leatham, 1950); $T$ inversion present in CR4 and in excess of that in CR7; depression of the S-T segment and T wave in IIIR.

In aortic valvular disease. All 20 cases in this group had either aortic stenosis, aortic incompetence, or both. Their routine cardiogram showed inversion of the T wave in lead I or in II and III, and in CR7. $\mathbf{R}$ was usually small and $\mathbf{S}$ large in lead III. 


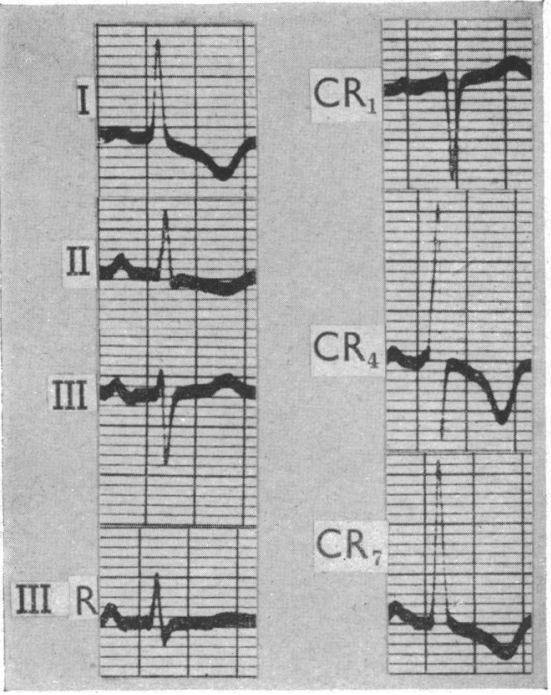

Fig. 9.-Hypertension with cardiac pain. Upright $\mathrm{T}$ in III, flat $\mathrm{T}$ in IIIR.
In six cases a $Q$ wave, less than $2 \mathrm{~mm}$. in depth, was present in III and it always persisted in IIIR. T III was upright in nine, flat in two, and inverted in nine. Of the nine with an upright $T$, it was lower in IIIR in five, and the $S-T$ segment was depressed without such a change in the $T$ in the other four. In the two instances of an upright or flat $T$ in III it became inverted in IIIR (Fig. 10) while in the nine in whom the $T$ was inverted in III the inversion was increased in IIIR in every instance.

It is not yet known for certain whether such changes indicate underlying coronary arterial disease or whether they are the outcome of a shift of the hypertrophied muscular mass, but this latter explanation is more likely because changes in IIIR may be the first cardiographic sign of aortic stenosis.

\section{Right Ventricular Preponderance}

Lead IIIR was examined in 10 patients with right heart hypertrophy either from pulmonary or from congenital heart disease; it showed no constant change in that the inverted $\mathrm{T}$ wave in lead III was sometimes unaltered in IIIR, sometimes less inverted and at other times more inverted, and this last change was invariable in the presence of digitalization.

\section{Left Bundle BRanch Block}

Lead IIIR was examined in 20 patients with left bundle branch block. Although it was not possible to name the cause of the block in all of them, hypertension was present in four and eight had chest pain reminiscent of cardiac pain. Among the unimportant changes in IIIR were a taller $\mathbf{R}$ wave and a shorter $\mathbf{S}$ than in lead III. The most noticeable change was a depression of the S-T segment, which took place in all save two cases; this S-T depression was not, however, associated with a lowering of $\mathrm{T}$ except in two patients with cardiac pain where an upright $T$ wave was depressed in one and inverted in another, inferring the presence of coronary arterial disease and cardiac infarction. In nine patients, a $Q$ wave, usually prominent, was present in lead III, but in seven of these its absence in IIIR proved it to be a false Q; in the other two, both of whom had cardiac pain, the $Q$ persisted (Fig. 11).

\section{Right Bundle Branch Block}

Lead IIIR was examined in 20 patients with right bundle branch block-8 with cardiac pain, 3 with auricular septal defect, 1 with pulmonary embolism, 1 with mitral stenosis, 1 with hypertension, and 6 without other evidence of heart disease. A common change in IIIR was a lowering of the R1 wave, and it was found in 12 out of 20 cases (Fig. 12). When changes took place in T III, which was inverted in 17 cases, it usually became less inverted irrespective of the cause of the block; in two it actually became upright.

In two patients, both subject to cardiac pain, the S-T segment became depressed. In three of the eight with cardiac pain, a $Q$ wave which had been absent in III developed in IIIR, and this provided the only sign of coronary arterial disease in these cases; in two others a $Q$ wave of less than $2 \mathrm{~mm}$. in lead III, persisted in IIIR, while in the remaining two, Q was absent in both leads III and IIIR. Among the 12 cases of right bundle branch block without cardiac pain a Q III was present in three and in each it was absent in IIIR.

\section{CARDIAC INFARCTION}

Lead IIIR was next examined in 77 patients with cardiac infarction. In 20 of these $T$ was inverted in leads I and CR4 placing the infarct anteriorly. In another 20, T was inverted in lead I and CR7 so that the infarct was disposed laterally, while in 37 patients the infarct was situated posteriorly and the T was inverted in lead III and often in lead II. The changes in IIIR will now be described for each of the three groups. 


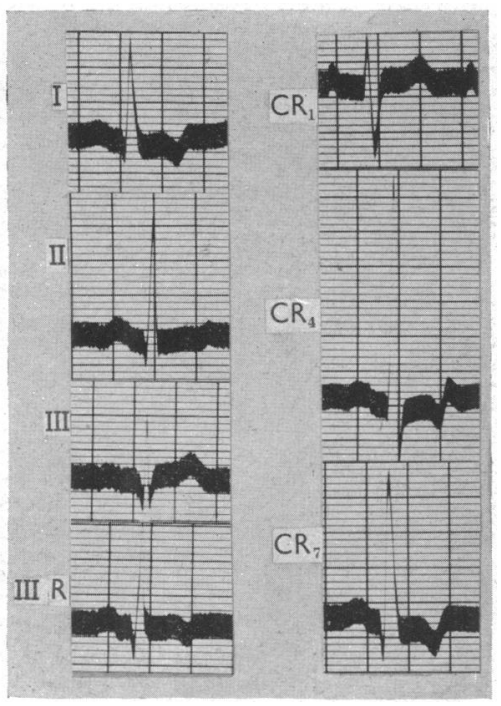

FIG. 10

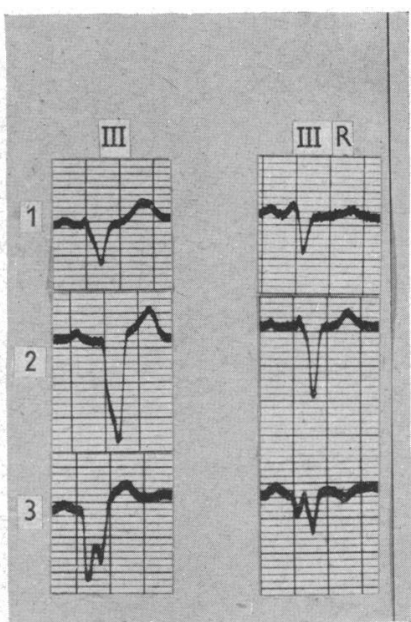

Fig. 11

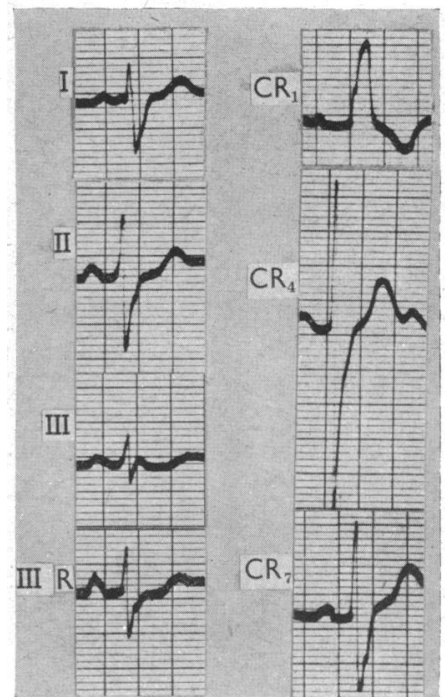

FIG. 12

FIG. 10.-Aortic stenosis. Upright $\mathrm{T}$ in III, inverted in IIIR.

Fig. 11.-Left bundle branch block. Common changes in IIIR shown as depression of S-T segment in Case 1, $Q$ wave disappearing in Case 2, and $Q$ remaining in Case 3.

Fig. 12.-Right bundle branch block. $R$ and early part of S-T segment in III depressed in IIIR.

In anterior cardiac infarction. In 20 patients where deformity of the $\mathrm{T}$ wave was confined to leads I and CR4, no significant change was found in IIIR. Naturally, the R wave often became taller and the $\mathrm{S}$ wave shorter in IIIR, but the $\mathrm{S}-\mathrm{T}$ segment never showed depression and there was no lowering of the upright $\mathrm{T}$ wave. In other patients (not included in this series) where the cardiographic signs of anterior infarction were uppermost, if depression of the S-T segment, and/or the T wave occurred in IIIR, the $T$ wave was also found to be low in CR7, showing that the infarction, although mostly in the front of the heart, was also extending laterally.

In lateral cardiac infarction. In 20 patients with inversion of $\mathrm{T}$ in leads I and CR7, the upright T III was invariably lower in IIIR and in one it became inverted. In 15 out of the 20 cases the S-T segment was depressed in addition to the lowered $T$ wave (Fig. 13).

In posterior cardiac infarction. In 37 patients where cardiac infarction was posterior according to the limb leads, Q III was deep in 23 , less than $2 \mathrm{~mm}$. in five, and absent in nine. In the first group Q IIIR remained prominent and greater than $2 \mathrm{~mm}$. in 20 , and persisted, but less than $2 \mathrm{~mm}$., in 3 cases; in the second group of 5 cases with a small Q III, it was absent in IIIR in three; in the third group of 9 cases the $Q$ remained absent in IIIR. T in IIIR was not once raised significantly in the 37 patients in all of whom it had been inverted in lead III. The S-T segment was depressed in IIIR in eight cases and in two of these Q III had been less than $2 \mathrm{~mm}$., while it was absent in the other six; the S-T segment was not depressed in IIIR in the 23 examples where Q III was greater than $2 \mathrm{~mm}$.

The incidence of S-T depression in IIIR and of T inversion in CR7 showed that cases hitherto nominated as posterior cardiac infarction from inspection of limb leads belonged to one of two groups, the TIII T7 kind and the QTIII kind.

Thus, in the TIII T7 kind (postero-lateral infarction) a significant $\mathrm{Q}$ was absent and $\mathrm{T}$ III was inverted, the S-T segment was depressed in IIIR, and this and the T wave were similarly deformed in CR7 (Fig. 14). In the QTIII kind (postero-inferior infarction) a significant $Q$ accompanied the $T$ inversion in lead III; there was no depression of the S-T in IIIR although the $T$ failed to rise, and neither the S-T segment nor the $T$ wave were affected in CR7 (Fig. 15). 


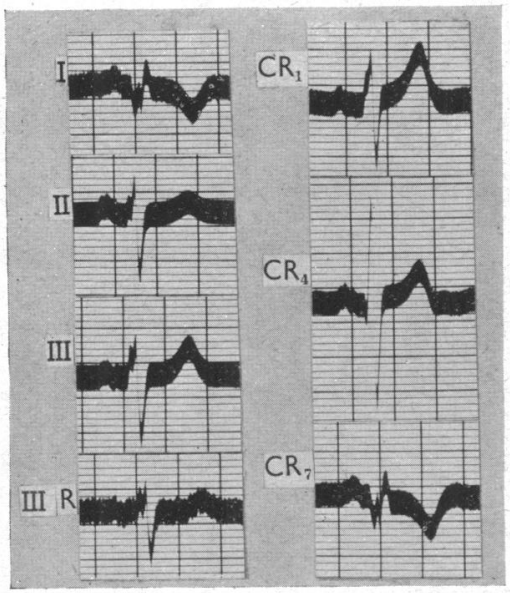

Fig. 13

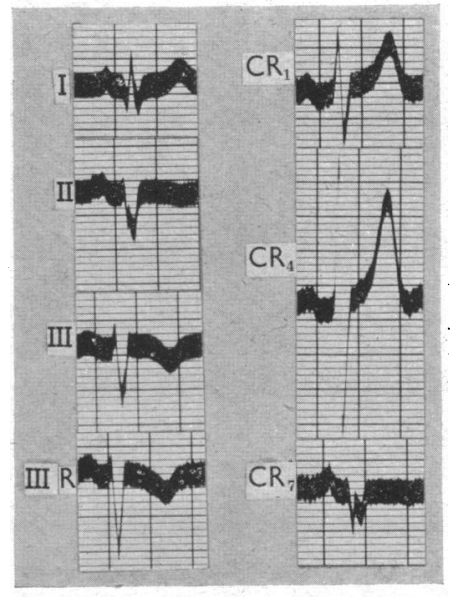

Fig. 14

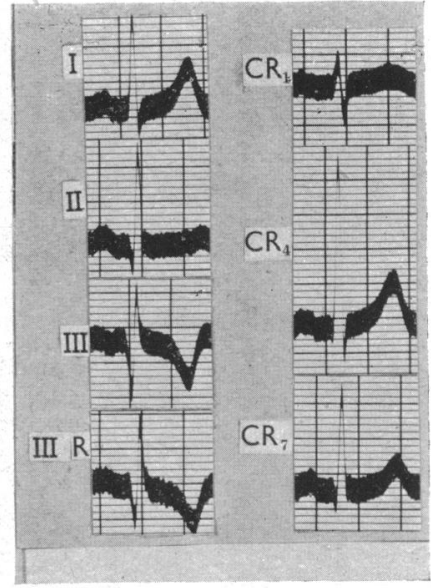

Fig. 15

Fig. 13.-Cardiac infarction (lateral.) Upright $\mathrm{T}$ in III lowered in IIIR.

FIG. 14.-Cardiac infarction (postero-lateral). Absent $Q$ in III, flat T in CR7, and S-T depression in IIIR.

FIG. 15.-Cardiac infarction (postero-inferior). - Significant $Q$ in III, upright $T$ in CR7, and no S-T depression in IIIR.

If the S-T or T in CR7 in this group showed a deformity, lead I and/or CR4 showed a similar change, or sometimes the $\mathrm{T}$ was sharply inverted in lead II, and the infarction was not confined to the lateral and posterior aspects of the heart.

Changes in lead IIIR as a lone sign of cardiac infarction. After determining the appearance of the IIIR cardiogram in health and the way it differed from lead III, and having discovered its pattern in several forms of heart disease, it was natural to test it in the diagnosis of coronary arterial disease when changes were absent in the customary limb and chest leads. Abnormal signs in IIIR were also sought in patients in whom the orthodox cardiogram produced only equivocal signs. Indeed,

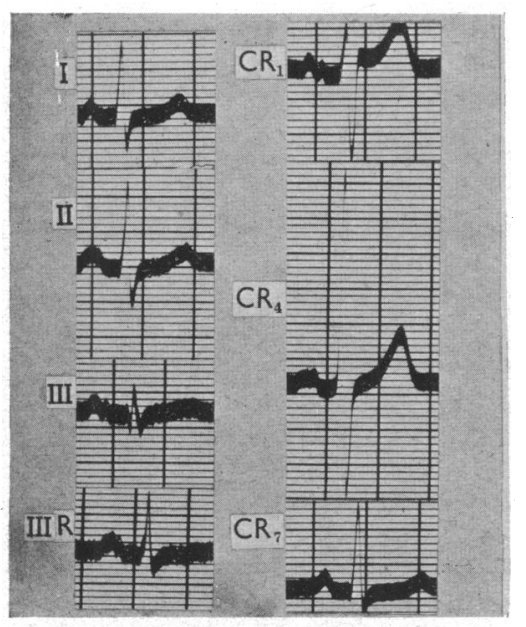

Fig. 16

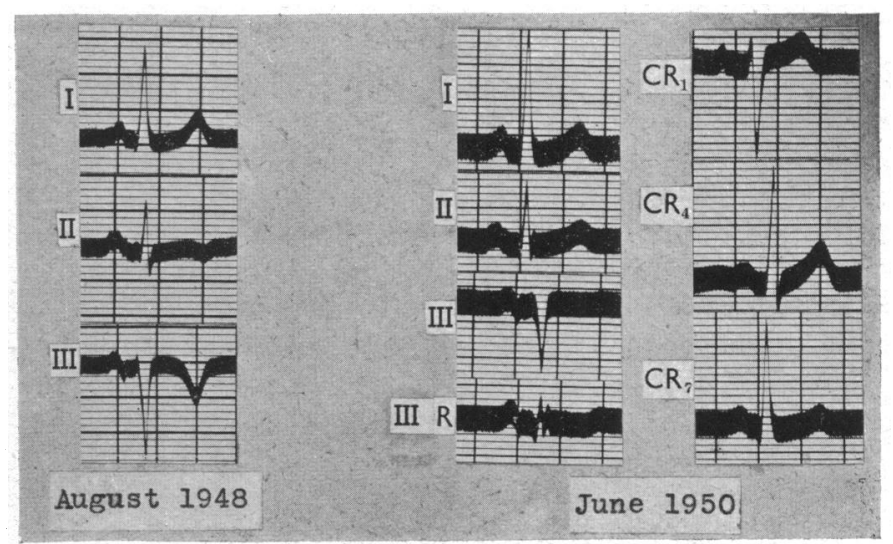

FIG. 17

FIG. 16. - Cardiac infarction. S-T depression in IIIR in patient with cardiac pain who died in an attack two months later.

Fig. 17.-Cardiac infarction. S-T depression in IIIR a lone abnormality in second cardiogram. 
it was in such cases that lead IIIR proved most useful, so that sometimes it showed depression of the S-T segment as the only abnormal cardiographic sign early in a patient's illness (Fig. 16), and only later would it be joined by other and more obvious signs of cardiac infarction (Fig. 18). Another time when the cardiogram might show recovery from an obvious deformity of the $T$ wave in a patient with cardiac pain, changes in lead IIIR remained as the only evidence of myocardial damage (Fig. 17). More common was the routine cardiogram where equivocal changes were present and where IIIR supported the suggestion that such changes indicated cardiac infarction.

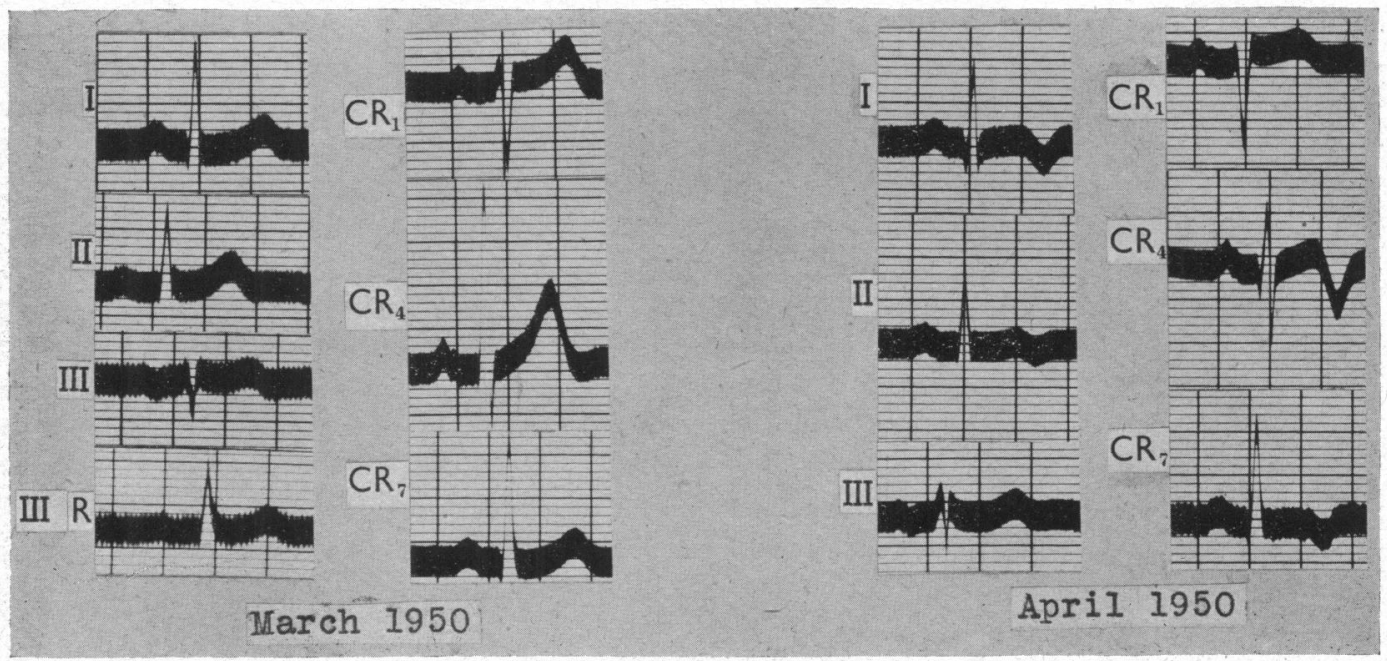

FIG. 18.-Cardiac infarction. S-T depression in IIIR a lone abnormality in first cardiogram.

\section{SUMmaRY AND CONCLUSIONS}

Variation in the pattern of lead III caused by a shift in the position of the heart from a change of body posture led to a study of the lead taken during deep inspiration in 200 healthy subjects and in the same number of patients with heart disease. Since respiration plays a part in the recording of this lead it has been named IIIR where $\mathbf{R}$ stands for respiration.

In no instance was the form of IIIR the same as III, giving proof of the unreliability of any lead that is built up in part from the electrical potential at the lower extremity. Among the common changes in IIIR in health were an increase in the height of the P, R, and T waves and a shortening of the S wave, compared with that found in lead III.

It was possible from the examination of lead IIIR in the healthy series to describe for it a code of normality in the adult. Thus, a $Q$ wave of $2 \mathrm{~mm}$. or under in lead III may persist in IIIR, but a $Q$ deeper than $2 \mathrm{~mm}$. is never found in IIIR. Again, a $Q$ in III never deepens in IIIR, nor appears in IIIR when absent in III. An upright $T$ wave in III never lessens in IIIR, while a flat or inverted $T$ rises significantly as a rule in IIIR unless, as rarely happens, the heart is prevented from descending during deep inbreathing, or when it even moves upwards (paradoxical displacement) as in paralysis of the left dome of the diaphragm. Depression of the S-T segment in IIIR is not a change so far found in healthy subjects.

In left ventricular preponderance from hypertension a depression of the $\mathrm{T}$ wave and $\mathrm{S}-\mathrm{T}$ segment was uncommon when cardiac pain was absent, but in those patients with pain such a change was invariable in IIIR; when this change took place in patients without pain it was regarded as evidence of added coronary arterial disease. In left ventricular preponderance from aortic stenosis depression of the S-T segment and/or of the $T$ wave in IIIR was a constant finding and this change may be the earliest cardiographic evidence of aortic stenosis. 
No conștant pattern was found for IIIR in the cases of right heart preponderance examined.

In left bundle branch block depression of the raised S-T segment in III took place in IIIR, but without noticeable lowering of the $T$ wave. A more significant change was the appearance of an $\mathbf{R}$ wave in IIIR which had often been absent in III, thereby showing that the Q wave had been deceptive; if no $R$ wave appeared it confirmed a diagnosis of cardiac infarction.

In right bundle branch block a reduction in amplitude of $\mathrm{R} 1$ was common while the $\mathrm{T}$ wave, usually inverted, became less so. Here again the value of IIIR was greatest when it proved the presence of a true $Q$ greater than $2 \mathrm{~mm}$. in lead III; or when a $\mathrm{Q}$, absent in III, made its appearance in IIIR, and in this event connoted the presence of coronary arterial disease.

Lead IIIR had its greatest use in the diagnosis of cardiac pain. In patients where the presence and site of cardiac infarction were told by the customary limb and chest leads, IIIR was found unchanged in anterior infarction, but the $S-T$ segment and the $T$ wave became depressed whenever the infarct was disposed laterally. Patients judged by the limb leads to have posterior infarction were placed in two groups in accordance with a cardiographic classification. In one (TIII T7 kind, postero-lateral infarction) inversion of T III was not associated with a significant $Q$ wave, and there was depression of the S-T segment and/or of the T wave in IIIR; similar changes also appeared in CR7. In the second (QTIII kind, postero-inferior infarction) a significant Q III was present in addition to $T$ inversion, and here no changes took place in IIIR except that the inverted $T$ failed to rise significantly.

In a number of patients lowering of the $S-T$ segment and the $T$ wave in IIIR provided the only cardiographic abnormality suggesting cardiac infarction, of which previous or subsequent tracings. gave proof. For this reason, lead IIIR should never be omitted from an electrocardiographic investigation of cardiac pain.

\section{REFERENCES}

Einthoven, W., Fahr., G., and de Waart, A. (1913). Pflüger's Arch. Physiol., 150, 275.

Lyle, A. M. (1944). Amer. Heart J., 28, 199.

Myers, G. B., and Oren, B. G. (1945). Amer. Heart J., 29, 708.

Norris, G. L., and Massie, E. (1947). Proc. Central Soc. Clin. Research, $20,57$.

Shillingford, J., and Leatham, A. (1950). Personal communication.

Videla, J. G. (1939). Rev. Argent Cardiol., 6, 146.

(1942). Variaciones Respiratorias del Electrocardiograma. Buenos Aires.

Waller, A. D. (1913). J. Physiol, 46, 57.

(1914). J. Physiol, 48, 40. 